In dieser neuen Rubrik wollen wir herausragende Publikationen von Mitgliedern der Gesellschaft für Kinder- und Jugendrheumatologie (GKJR) sowie andere interessante Artikel aus dem Bereich Kinderrheumatologie in Kurzform vorstellen.

\section{Assoziierte Lungen- erkrankung bei Patienten mit einer systemischen juvenilen Arthritis}

In den letzten Jahren wurde bei Patienten mit einer systemischen juvenilen Arthritis (sJIA) zunehmend eine assoziierte Lungenerkrankung (LD) beschrieben. In der vorliegenden Arbeit trugen Schulert et al. die Daten von 18 Patienten zusammen.

Schulert et al. Systemic juvenile idiopathic arthritis-associated lung disease: characterization and risk factors. Arthritis \& Rheumatology 2019;

11: 1943-1954

In den Röntgenuntersuchungen des Thorax zeigten sich dabei diffuse Verschattungen, subpleural retikuläre Verdichtungen, interlobärseptale Verdickungen und Lymphknotenvergrößerungen. Histopathologischer waren lymphoplasmazelluläre Infiltrate, Zeichen der pulmonal alveolären Proteino- se (PAP) und der lipoiden Pneumonie nachweisbar. Verglichen mit Patienten ohne Lungenbeteiligung wiesen die beschriebenen sJIA-LD Patienten signifikant differente Charakteristika auf: sie waren jünger bei Diagnosestellung, hatten häufiger Episoden eines Makrophagenaktivierungssyndroms (MAS) sowie häufiger Nebenwirkungen einer Biologikatherapie und höhere Interleukin-18-Spiegel. Dabei konnte ein Zusammenhang zu einer familiären oder primären PAP ausgeschlossen werden, da diese Patienten weder genetisch, serologisch noch funktionell Hinweise auf eine Dysfunktion des Granulozyten-Makrophagen-KoIonie stimulierenden Faktors aufwiesen. Darüber hinaus fand sich in der bronchoalveolären Lavage (BAL) nur selten proteinreiches Material sowie wenig lipidbeladene Makrophagen wie bei Patienten mit primärer PAP. Allerdings waren in der BAL hohe Spiegel von IL-18 und den Interferon-gamma induzierten Chemokinen CXCL9 und CXCL10 zu finden. Im Lungengewebe war mittels eines Genexpressionsprofils eine Hochregulierung des Typ-II-Interferons und des T-Zellaktivierungsnetzwerks nachweisbar. Da diese Befunde selbst in Gewebeproben von Patienten ohne histopathologisch nachweisbare Lungenveränderungen vorhanden waren, gehen die Autoren davon aus, dass diese „Aktivierungssignatur“ der LD vorangeht und sie weiter unterhält.

\section{FAZIT}

In dieser wichtigen Arbeit werden erstmals systematisch charakteristische Befunde einer sJIA-assoziierten Lungenerkrankung beschrieben. Wichtig ist sicherlich eine klinische Awareness für diese - offenbar zunehmende - schwerwiegende Erkrankung, die mit folgenden Risikofaktoren assoziiert war: Alter <2 Jahre bei Erkrankungsbeginn, rezidivierendes MAS, Nebenwirkungen auf Tocilizumab sowie hohe Interleukin18-Spiegel. Trommelschlegelfinger waren dabei eine der klinischen Hauptbefunde. Bei Hinweisen auf eine Lungenbeteiligung ist eine niederschwellige Diagnostik (Lungenfunktion, DLCO, 6-Minuten-Gehtest, HR-CT) anzuraten.

Um dieses Krankheitsbild weiter verfolgen zu können, ist eine detaillierte Meldung und Analyse in unseren vorhandenen Registern (Kerndokumentation und BIKER) wünschenswert.

Toni Hospach, Stuttgart 